\title{
Índice leucoglucémico como marcador pronóstico de complicaciones en pacientes con infarto agudo de miocardio
}

\section{Leukoglucemic index as a prognostic marker for complications in patients with acute myocardial infarction}

\author{
Paola L. Cuesta-Mero ${ }^{*}$, Laura A. García-Romo² y Álvaro D. Villacres-López ${ }^{3}$ \\ ${ }^{1}$ Postgrado de Medicina Interna, Universidad Central del Ecuador; 2 Servicio de Cardiología, Hospital Eugenio Espejo; ${ }^{3}$ Servicio de Cardiología \\ Hospital de los Valles. Quito, Ecuador
}

\begin{abstract}
Resumen
Introducción: El índice leucoglucémico es un factor pronóstico, poco conocido y utilizado, que relaciona la glucemia y los leucocitos registrados al ingreso hospitalario. Su importancia radica en la determinación del riesgo de complicaciones y la mortalidad en pacientes con infarto agudo de miocardio. Objetivo: Establecer al índice leucoglucémico como factor predictor de complicaciones durante la evolución intrahospitalaria en los pacientes con infarto agudo de miocardio. Método: Estudio de tipo epidemiológico, analítico, observacional y de corte transversal, en una cohorte de pacientes que ingresaron con infarto agudo de miocardio durante un período de un año y tres meses. Resultados: Se incluyó en el estudio a 205 pacientes con infarto agudo de miocardio, cuyo promedio de edad fue de $62.99 \pm 12.2$ años, con predominio en varones con $69.3 \%$. El infarto agudo de miocardio con elevación del segmento ST predominó en frecuencia con $63.9 \%$ y mayor tasa de complicaciones $(p<0.001)$. El promedio del índice leucoglucémico fue de 1,578.41 $\pm 1,208.1$ y el punto de corte establecido fue de 656.8, de acuerdo con la curva ROC, con sensibilidad del $95.8 \%$ y especificidad del $73 \%$ para la predicción de complicaciones intrahospitalarias, con OR de 7.89. Conclusión: Un índice leucoglucémico > 656.8 representa un riesgo de 7.89 veces de desarrollar complicaciones en la población estudiada.
\end{abstract}

Palabras clave: Infarto agudo de miocardio. Índice leucoglucémico. Pronóstico.

\section{Abstract}

Introduction: Leukoglycemic index is a poorly known and used prognostic factor that correlates blood glucose and leukocytes which are taken at hospital admission. Its importance lies in determining complications and mortality risks in patients with acute myocardial infarction. Objective: To establish the leukoglycemic index as a predictor of complications during the in-hospital evolution in patients with acute myocardial infarction. Methods: Epidemiological, observational, cross-sectional, crosssectional study on a cohort of patients admitted with acute myocardial infarction during a period of 1 year 3 months. Results: 205 patients with acute myocardial infarction were included in this study. The mean age was $62.99 \pm 12.2$ years old, more frequently in males by $69.3 \%$. It was determined that acute myocardial infarction with ST elevation predominated both in frequency with $63.9 \%$, as well as with a higher rate of complications $(p<0.001)$. The average of leukoglycemic index

\section{Correspondencia:}

*Paola L. Cuesta-Mero

E-mail: liscuestamero@ hotmail.es
Disponible en internet: 10-06-2021 Rev Colomb Cardiol. 2021;28(2):119-127 www.rccardiologia.com 0120-5633 / C 2020 Sociedad Colombiana de Cardiología y Cirugía Cardiovascular. Publicado por Permanyer. Este es un artículo open access bajo la licencia CC BY-NC-ND (http://creativecommons.org/licenses/by-nc-nd/4.0/). 
was $1578.41 \pm 1208.1$ and the cut-off point was 656.8 , according to ROC curve, with sensitivity of $95.8 \%$ and specificity of $73 \%$ for the prediction of inhospital complications, OR 7.89. Conclusions: A leukoglycemic index greater than 656.8 represents a 7.89-fold risk of developing complications in the study population.

Key words: Acute myocardial infarction. Leukoglucemic index. Prognosis.

\section{Introducción}

El infarto agudo de miocardio constituye un gran problema de salud pública no sólo por el alto costo económico que genera su atención, sino también por la elevada mortalidad que causan sus complicaciones. Se define como la necrosis de miocardiocitos en un contexto clínico consistente con isquemia miocárdica aguda'. Se presenta con mayor frecuencia en la sexta y séptima décadas de la vida y es la causa individual más común de muerte mundial, con un $12.8 \%$, la cual depende de factores como edad, tratamiento, infartos previos, territorio de isquemia, diabetes, entre otros.

En los últimos años ha cobrado mayor importancia el papel de la inflamación en el desarrollo de aterosclerosis y la patogenia de la trombosis coronaria. Estudios recientes han comprobado que la elevación de marcadores inflamatorios, como proteína $\mathrm{C}$ reactiva $\mathrm{e}$ interleucinas, en el contexto del paciente con infarto agudo de miocardio se relaciona con un mayor número de complicaciones ${ }^{1}$. Sin embargo, estas pruebas tienen como principales limitantes el alto costo y la poca accesibilidad, sobre todo en los países en vías de desarrollo, en los cuales la falta de recursos técnicos y económicos son problemas transcendentales en los servicios de salud.

Se sabe que el valor de la glucemia y los leucocitos, por sí solo, expresa el proceso inflamatorio y metabólico observado en el infarto agudo de miocardio. Sin embargo, la interrelación de ambos parámetros, conocido como índice leucoglucémico, se considera un marcador predictor de complicaciones durante la estancia hospitalaria y ha generado nuevas expectativas en la práctica médica desde el 2010, cuando un grupo de investigadores lo utilizó para estratificar a los pacientes con infarto agudo de miocardio con elevación del segmento ST al aplicar la fórmula: índice leucoglucémico = glucemia $(\mathrm{mg} / \mathrm{dl}) \mathrm{x}$ leucocitos (células)/1,000. Así lograron identificar que aquéllos con índice leucoglucémico $>1,600$ presentaban una tasa de complicaciones del $61 \%$ contra el $19 \%$ que generó el valor $<1,600^{2}$. En otro trabajo se aplicó este índice en 128 pacientes con infarto de miocardio con elevación del ST, en el que establecieron que un punto de corte $>1,158$ se relacionaba con mayor mortalidad y complicaciones ${ }^{3}$. Como se observa, aún no se encuentra definido el punto de corte del índice leucoglucémico. Existe poca información acerca de éste en el infarto de miocardio con elevación del ST, y menos aún en pacientes con infarto agudo de miocardio sin elevación del ST, sin que se conozca su importancia, a pesar de que podría ser de valiosa utilidad en la práctica médica.

El objetivo del estudio es establecer el índice leucoglucémico como factor predictor de complicaciones durante la evolución intrahospitalaria en los pacientes con infarto agudo de miocardio.

\section{Método}

Estudio epidemiológico, analítico y observacional sobre una cohorte de pacientes con infarto agudo de miocardio, ingresados a la unidad de coronarios durante el período de marzo del 2015 a mayo del 2016, cuyo número final fue 205. El estudio tuvo aprobación del comité ético del hospital seleccionado. Se incluyó a hombres y mujeres $\geq 18$ años con infarto agudo de miocardio con y sin elevación del segmento ST, diabéticos y no diabéticos. Se excluyó a los pacientes con enfermedades infecciosas conocidas y documentadas al ingreso hospitalario, enfermedades hematológicas antiguas y actuales, inmunodeficiencias conocidas y documentadas, y aquellos en quienes faltaron valores de glucemia y leucocitos en las primeras 24 horas del ingreso.

Las variables analizadas fueron género, edad, comorbilidades, índice leucoglucémico, diabetes mellitus, tipo de infarto, topografía del infarto, tratamiento del infarto, complicaciones intrahospitalarias, muerte, calificaciones TIMI y GRACE, y días de hospitalización.

De las variables cualitativas se tomaron las frecuencias absoluta y relativa. Con las variables cuantitativas se usaron valores de tendencia central y dispersión. El grado de relación entre las variables cualitativas se determinó mediante ji cuadrada. Se utilizó el análisis de curva ROC para establecer el punto de corte del índice leucoglucémico $(p<0.05)$. 
Tabla 1. Características epidemiológicas de los pacientes con infarto agudo de miocardio de acuerdo con el género

\begin{tabular}{|l|c|c|}
\hline $\mathbf{n}=\mathbf{2 0 5}$ & Hombre $(\mathbf{n}=142)$ & Mujer $(\mathbf{n}=63)$ \\
\hline Edad & $61.55 \pm 11.2$ años & $66.24 \pm 13.9$ años \\
\hline IAM & $69.26 \%$ & $30.73 \%$ \\
\hline HTA & $73(51.4 \%)$ & $48(76.2 \%)$ \\
\hline DM & $31(21.8 \%)$ & $24(38.1 \%)$ \\
\hline $\begin{array}{l}\text { Cardiopatía } \\
\text { isquémica previa }\end{array}$ & $38(26.8 \%)$ & $13(20.6 \%)$ \\
\hline Tabaquismo & $84(59.2 \%)$ & $11(17.5 \%)$ \\
\hline Alcoholismo & $79(55.6 \%)$ & $9(14.3 \%)$ \\
\hline Leucocitos & 10.595 células & 10.182 células \\
\hline Glucemia & $142 \mathrm{mg} / \mathrm{dl}$ & $148.48 \mathrm{mg} / \mathrm{dl}$ \\
\hline ILG & $1567.8 \mathrm{mg} / \mathrm{dl} x$ & $1602.27 \mathrm{mg} / \mathrm{dl} x$ \\
\hline
\end{tabular}

DM: diabetes mellitus; HTA: hipertensión arterial; IAM: infarto agudo de miocardio; ILG: índice leucoglucémico.

\section{Resultados}

Se incluyó a 205 pacientes, cuyo promedio de edad fue de $62.99 \pm 12.2$ años, con un intervalo de 28 a 91 años (Tabla 1). En la figura 1 se observa que el tipo de infarto más común fue el infarto agudo de miocardio con elevación del segmento ST, con un $63.9 \%(n=131)$, y al compararlo con el género se encontró una diferencia estadísticamente significativa; proporcionalmente, los varones padecen más infarto agudo de miocardio con elevación del segmento ST que las mujeres, $\chi^{2}$ $(1,5.23), p=0.022$.

Al comparar el tipo y la topografía del infarto se encontró que, en términos proporcionales, los de la cara lateral son más similares al infarto agudo de miocardio $\sin \mathrm{ST}, \chi^{2}(4,21.78), \mathrm{p}<0.001$ (Fig. 2).

El $67.8 \%(n=139)$ de los pacientes sufrió un infarto agudo de miocardio con KILLIP I al momento de su valoración; sólo el $1 \%(n=2)$ tenía un KILLIP de IV (Fig. 3). Al correlacionar la escala GRACE con el índice leucoglucémico no se encontraron diferencias significativas (Fig. 4). El riesgo TIMI tuvo un puntaje promedio de $4.0 \pm 2.35$, con una mediana y moda de 3 , y un máximo de 13 puntos. El $25 \%$ de los pacientes con infarto agudo de miocardio mostró un TIMI > 5 puntos.

Asimismo, se determinó que la mortalidad es mayor en el infarto con elevación del segmento ST (Tabla 2). Y al comparar el número de complicaciones

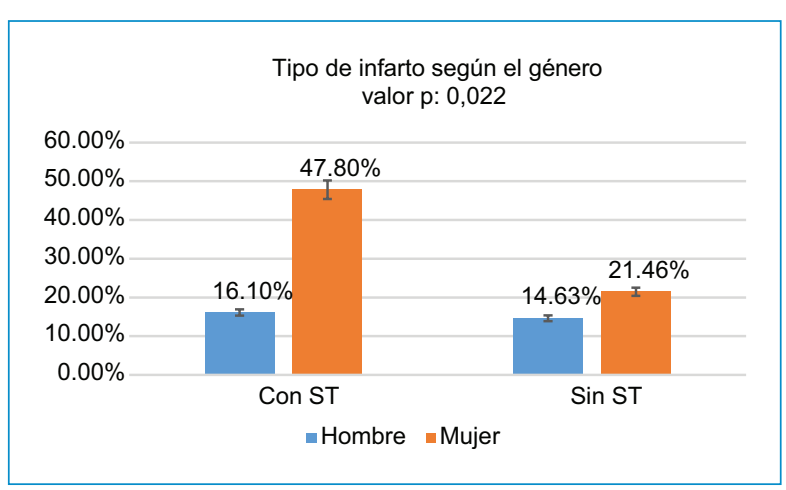

Figura 1. Comparación según el género del tipo de infarto agudo de miocardio.

IAM con ST: Infarto agudo de miocardio sin elevación del segmento ST; IAM sin ST: Infarto agudo de miocardio sin elevación del segmento ST.

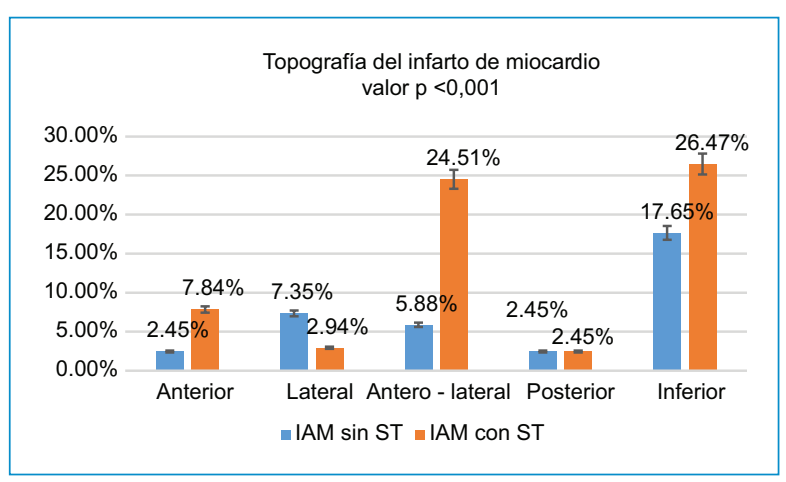

Figura 2. Topografía (localización) según el tipo de infarto agudo de miocardio.

IAM con ST: Infarto agudo de miocardio sin elevación del segmento ST, IAM sin ST: Infarto agudo de miocardio sin elevación del segmento ST.

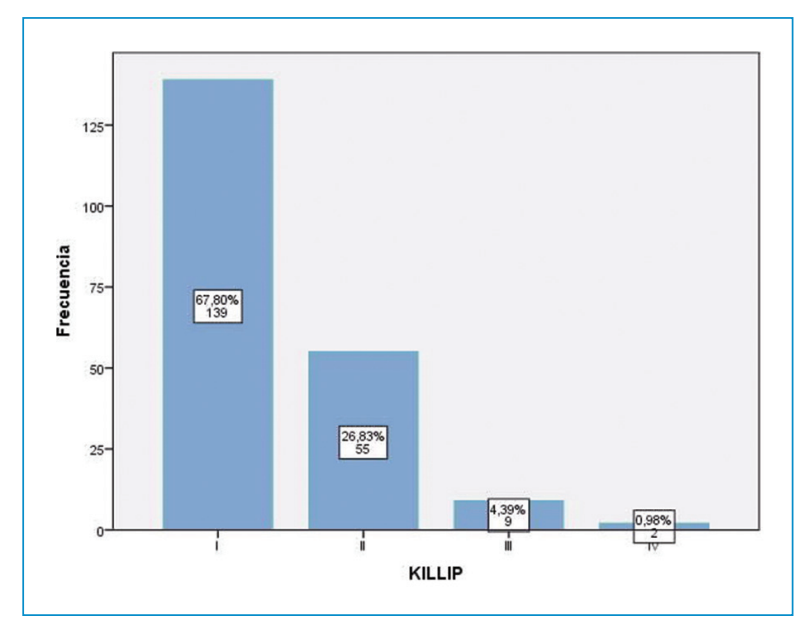

Figura 3. Distribución de frecuencia según la clasificación KILLIP. 
Tabla 2. Complicaciones y mortalidad de acuerdo con el tipo de infarto en pacientes con infarto agudo de miocardio

\begin{tabular}{|c|c|c|c|}
\hline$n=205$ & IAM con ST & IAM sin ST & $x^{2}$ \\
\hline ICC & $30.5 \%(n=40)$ & $14.9 \%(n=11)$ & $\chi^{2}(1,6.21) p<0.01$ \\
\hline FA & $12.2 \%(n=16)$ & $2.7 \%(n=2)$ & $\chi^{2}(1,5.34) p<0.02$ \\
\hline Arritmia ventricular & $9.9 \%(n=13)$ & $5.4 \%(n=4)$ & $\chi^{2}(1,1.26)$ p 0.26 \\
\hline Bloqueo AV & $6.1 \%(n=8)$ & $2.7 \%(n=2)$ & $\chi^{2}(1,1.18)$ p 0.27 \\
\hline Reinfarto & $0.8 \%(n=1)$ & $5.4 \%(n=4)$ & $\chi^{2}(1,4.81) p<0.03$ \\
\hline Choque & $19.8 \%(n=26)$ & $6.8 \%(n=5)$ & $\chi^{2}(1,6.31) p<0.01$ \\
\hline Angina luego del AM & $45.8(n=60)$ & $39.2 \%(n=29)$ & $\chi^{2}(1,0.84)$ p 0.36 \\
\hline Mortalidad & $14.5 \%(n=19)$ & $6.8 \%(n=5)$ & $\chi^{2}(1,2.74)$ p 0.09 \\
\hline
\end{tabular}

AV: auriculoventricular; FA: fibrilación auricular; IAM: infarto agudo de miocardio; IC: insuficiencia cardíaca.

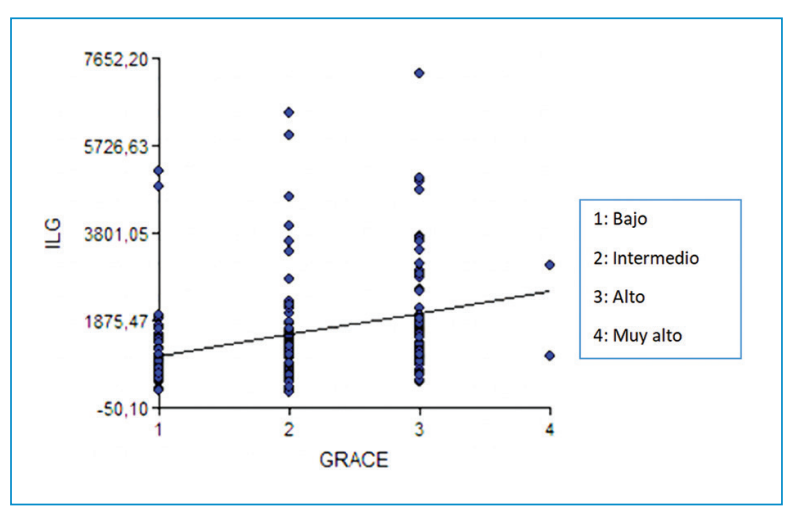

Figura 4. Correlación entre índice leucoglucémico y Score GRACE.

GRACE: global registry of acute coronary events.

de acuerdo con el tipo de infarto, en éste se encontró una diferencia significativa ya que hay mayor número de complicaciones $(p<0.001$, prueba de $U$ de Mann-Whitney).

Respecto del índice leucoglucémico, al cotejarlo con las características clínicas se encontró que los pacientes con infarto agudo de miocardio con elevación del ST y complicaciones por infarto tienen en promedio un índice leucoglucémico más elevado, diferencia que es estadísticamente significativa (Tabla 3).

La glucemia y el índice leucoglucémico fueron significativamente más altos en los diabéticos que en los no diabéticos (Tabla 4). Al comparar los leucocitos, la glucemia y el índice leucoglucémico, según los tipos de infarto, se encontró que éstos tenían diferencias estadísticamente significativas (Tabla 5).

Al correlacionar el índice leucoglucémico con el riesgo calculado por GRACE se identificó que esta relación es de tipo positiva y estadísticamente significativa: a medida que se incrementa el índice leucoglucémico, aumenta el riesgo determinado por GRACE, con un coeficiente de correlación de Sperman de +0.45 $(n=205, p<0.001)$. $Y$ al compararlo con el riesgo calculado por TIMI se observó que esta relación también era positiva y estadísticamente significativa: a medida que aumenta el índice leucoglucémico, también lo hace el riesgo determinado por TIMI, con un coeficiente de correlación de Sperman de $+0.34(n=205$, $\mathrm{p}<0.001$ ).

Para evaluar el valor predictivo del índice leucoglucémico en relación con la mortalidad se elaboró una curva ROC; el punto de corte del índice leucoglucémico con mayor discriminación fue 851.60 y el área bajo la curva fue de 0.64 (IC95\%, 0.53-0.75), con una sensibilidad del $95.8 \%$ y especificidad del $68 \%$ (Fig. 5).

Para evaluar el valor predictivo del índice leucoglucémico en relación con las complicaciones se trazó una curva ROC; el punto de corte del índice leucoglucémico con mayor discriminación fue de 656.8, con sensibilidad del $95.8 \%$ y especificidad del $73 \%$, y área bajo la curva de 0.80 (IC95\%, 0.73-0.87) (Fig. 6).

Con el punto de corte del índice leucoglucémico (656.8) se calculó el riesgo de complicaciones con un valor superior, el cual es de una razón de disparidad (OR) de 7.89 (IC95\%, 2.91-21.35). Proporcionalmente, los pacientes con infarto agudo de miocardio que ingresan con un índice leucoglucémico > 656.8 tienen 7.89 veces más posibilidades de mostrar complicaciones, diferencia que es estadísticamente significativa, $\chi^{2}(1,20.69), p<0.001$ (Fig. 7). 
Tabla 3. Índice leucoglucémico de acuerdo con las características clínicas y complicaciones en pacientes con infarto agudo de miocardio

\begin{tabular}{|l|c|c|c|}
\hline $\mathbf{n}=\mathbf{2 0 5}$ & \multicolumn{2}{|c|}{ Promedio y desviación estándar (por grupo) } & \multicolumn{1}{c|}{ Prueba t de Student } \\
\hline Género & H: $1567.83 \pm 1170.07$ & M:1602.27 \pm 1299.3 & $\mathrm{p}=0.85$ \\
\hline IAM con ST & Sí: $1820.44 \pm 1353.5$ & No: 1139.35 & $\mathrm{p}<0.001$ \\
\hline Complicaciones & Sí: $1797.12 \pm 12.15$ & No: $1084.94 \pm 1050.62$ & $\mathrm{p}<0.001$ \\
\hline LRA & Sí: $1854.9 \pm 1370$ & No: $1536.28 \pm 1151.28$ & $\mathrm{p}=0.27$ \\
\hline Muerte & Sí: $1912.02 \pm 1194.17$ & No: $1534.18 \pm 1206.31$ & $\mathrm{p}=0.15$ \\
\hline
\end{tabular}

IAM con ST: infarto agudo de miocardio con elevación del ST; LRA: lesión renal aguda.

Tabla 4. Comparación de leucocitos, glucemia e índice leucoglucémico de acuerdo con el tipo de infarto en pacientes con infarto agudo de miocardio

\begin{tabular}{|l|c|c|c|}
\hline $\mathbf{n}=205$ & Sin ST & Con ST & Prueba $\mathbf{t}$ de Student \\
\hline Leucocitos $\mathrm{mm}^{3}$ & $9213.23 \pm 2988.42$ & $11176.95 \pm 347.39$ & $\mathrm{p}<0.001$ \\
\hline Glucemia mg/dl & $118.82 \pm 46.158$ & $158.48 \pm 98.24$ & $\mathrm{p}<0.001$ \\
\hline ILG mg/dl x células & $1139.35 \pm 714.57$ & $1826.44 \pm 1353.5$ & $\mathrm{p}<0.001$ \\
\hline
\end{tabular}

ILG: índice leucoglucémico.

Tabla 5. Comparación de leucocitos, glucemia e índice leucoglucémico de acuerdo con diabetes mellitus en pacientes con infarto agudo de miocardio

\begin{tabular}{|l|c|c|c|}
\hline $\mathrm{n}=\mathbf{2 0 5}$ & Diabéticos & No diabéticos & Prueba $\mathrm{t}$ de Student \\
\hline Leucocitos mm & $10255.8 \pm 3351.72$ & $1054.93 \pm 3437.15$ & $\mathrm{p}=0.59$ \\
\hline Glucemia $\mathrm{mg} / \mathrm{dl}$ & $213.11 \pm 131.69$ & $118.89 \pm 35.77$ & $\mathrm{p}<0.001$ \\
\hline ILG $\mathrm{mg} / \mathrm{dl} x$ células & $2342.51 \pm 1809.97$ & $1298.25 \pm 718.71$ & $\mathrm{p}<0.001$ \\
\hline
\end{tabular}

ILG: índice leucoglucémico.

\section{Discusión}

En este trabajo se intenta reconocer la utilidad del índice leucoglucémico como predictor de complicaciones en pacientes con infarto agudo de miocardio, al establecer su importancia en los resultados obtenidos (OR, 7.89; IC95\%, 2.91-21.35; $p<0.001$ ), independientemente del tipo de infarto. En los resultados que publicaron Quiroga, et al. ${ }^{2}$, en un estudio con 101 pacientes, el índice leucoglucémico elevado en sujetos ingresados con infarto agudo de miocardio con elevación del ST se correlacionó con mortalidad y complicaciones (OR, 6.2; IC95\%, 2.65-15.55; $\mathrm{p}<0.0002$ ).
El fundamento fisiopatogénico que explica la correlación establecida por el índice leucoglucémico lo determinan dos factores; por un lado, la hiperglucemia de estrés secundaria a la resistencia a la insulina causada por inhibición de GLUT-4, que afecta la producción de óxido nítrico y favorece la vasoconstricción, e incrementa la generación de radicales libres que entorpecen la función plaquetaria y la fibrinólisis, lo que acentúa la obstrucción del flujo sanguíneo y perpetúa la isquemia 4 . Y, por otro lado, la leucocitosis expresa el proceso inflamatorio observado en la aterosclerosis y que no sólo influye en la desestabilización de la placa, sino también en la hipercoagulabilidad 


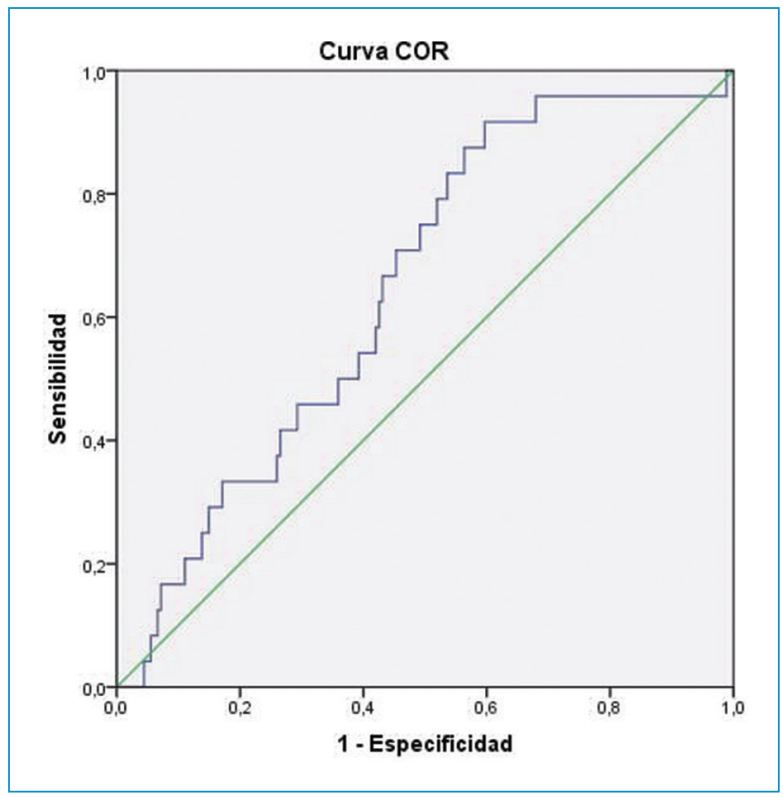

Figura 5. Curva COR para el índice leucoglicémico y mortalidad por infarto agudo de miocardio. COR: característica operativa del receptor.

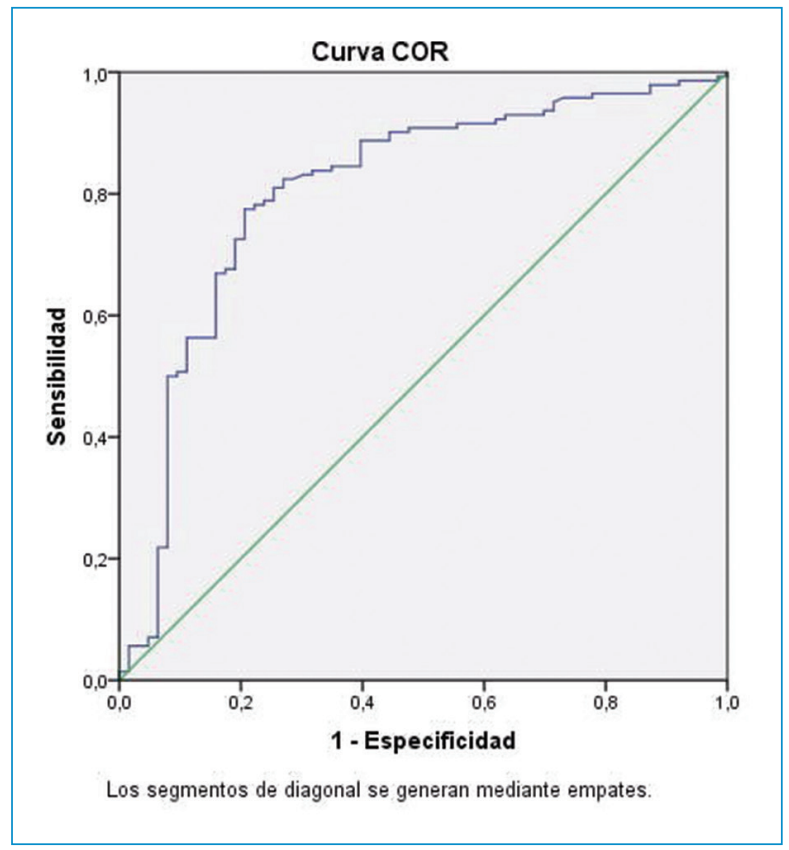

Figura 6. Curva ROC para índice leucoglucémico y complicaciones en pacientes con infarto agudo de miocardio.

que empeora la obstrucción coronaria. La necrosis miocárdica desencadena inflamación, lo cual agrava el problema ${ }^{5}$.

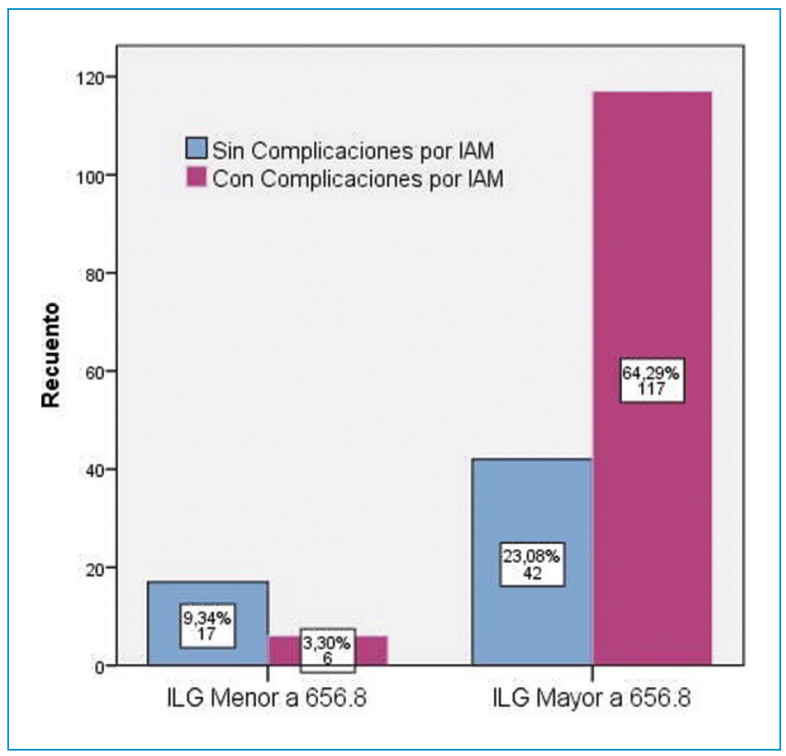

Figura 7. Comparación del ILG (corte mayor o menor de 656.8 ) y presencia de complicaciones por infarto agudo de miocardio leucoglicémico.

ILG: Índice Leucoglucémico.

El punto de corte del índice leucoglucémico como predictor de complicaciones para la población estudiada fue de 656.8, con una sensibilidad y especificidad del $95.8 \%$ y $73 \%$, respectivamente (IC95\%, 0.73-0.87, área bajo la curva de 0.8). Con dicho valor, el riesgo de complicaciones en el paciente infartado es de 7.89 veces mayor, lo cual difiere de los estudios establecidos con anterioridad; Quiroga, et al. ${ }^{2}$ definieron como punto de corte un valor de $1.600(p<0.0002)$ y establecieron diferencias estadísticamente significativas respecto de resultados menores. La tasa de complicaciones de los pacientes con infarto de miocardio con elevación del ST con un índice leucoglucémico $<1.600$ fue del $19 \%$, y de $61 \%$ cuando el índice fue $>1.600$. En este caso se atribuye la diferencia en relación con este estudio a varios motivos: a) la población investigada fue menor que la del presente trabajo, 101 contra 205 pacientes; b) se emplearon valores de leucocitos tomados hasta $48 \mathrm{~h}$ posteriores al ingreso respecto del recuento de leucocitos considerado a la admisión hospitalaria; c) el porcentaje de diabéticos era de $12 \%$, mientras que en el estudio actual es del $26.8 \%$, con base en la premisa de que los pacientes diabéticos poseen mayor riesgo de complicaciones ${ }^{6}$. Por otra parte, León-Aliz et al. ${ }^{3}$ establecieron como punto de corte el valor $>1.158$, con un área bajo la curva de 0.682 (IC95\%, 0.590-0.775), significativamente menor 
respecto de este estudio. Reyes, et al. realizaron un estudio con 142 pacientes con infarto de miocardio con y sin elevación del ST y un punto de corte similar al que expusieron Quiroga et al., pero al valorar el área bajo la curva es menor a la presentada en este trabajo (0.63 vs 0.8).

Esta investigación señala que el índice leucoglucémico como predictor de mortalidad tiene un punto de corte de 851.60, con área bajo la curva de 0.64 (IC95\%, 0.53-0.75), sensibilidad del $95.8 \%$ y especificidad del $68 \%$. En los estudios mencionados no se establecen diferencias respecto del punto de corte para complicaciones y mortalidad; sin embargo, los datos estadísticos en este estudio (área bajo la curva e intervalo de confianza) son similares a los de León-Aliz et al., expuestos con anterioridad. La versatilidad de los resultados podría deberse a que la variable muerte se tomó como parte de las complicaciones en las investigaciones referidas, a diferencia del presente estudio en el que se obtuvo de manera separada.

Los pacientes infartados tuvieron puntajes de GRACE y TIMI altos, de $32.2 \%$ y $25 \%$ respectivamente, lo cual se relaciona con mayor riesgo de morbimortalidad $^{7-9}$. Es importante destacar que el objetivo de la investigación era establecer la relación existente entre el índice leucoglucémico y dichos puntajes, cuyo resultado mostró una relación directamente proporcional, pues a medida que se incrementa el índice leucoglucémico también lo hace el riesgo calculado por GRACE y TIMI, con un coeficiente de correlación de 0.45 y 0.34 , respectivamente. No existen artículos que describan la interrelación entre estos parámetros; por lo tanto, este estudio es el que la establece por primera vez. Sí se ha descrito, en cambio, la relación de hiperglucemia de estrés, con la puntuación de TIMI y GRACE en infarto de miocardio, la cual es directamente proporcional $^{10}$.

De manera adicional se encontró que existe diferencia del valor del índice leucoglucémico en el infarto agudo de miocardio con y sin elevación del segmento ST, ya que se determinó que los pacientes con infarto agudo de miocardio y elevación del segmento ST tienen un índice leucoglucémico mayor, diferencia que es estadísticamente significativa $(p<0.001)$. Sin embargo, se podría explicar desde el punto de vista fisiopatológico debido a que el infarto agudo de miocardio con ST elevado se produce por oclusión completa de una arteria coronaria, lo que lleva a la isquemia y posterior necrosis, mientras que en el infarto agudo de miocardio sin elevación la obstrucción es parcial y por tanto el compromiso del miocardio es menor?.
También se encontró que el índice leucoglucémico en pacientes diabéticos es mayor, dado que la hiperglucemia de estrés, independiente de la existencia de diabetes, se relacionó con la aparición de episodios adversos mayores como muerte, reinfarto e ictus en el infarto de miocardio ${ }^{10}$. Además, León-Aliz et al. ${ }^{3}$ analizaron los principales desenlaces del estudio (muerte, complicaciones y éxito de la trombólisis), en pacientes diabéticos y no diabéticos, y encontraron que los valores de índice leucoglucémico fueron significativamente superiores respecto de aquellos pacientes que tuvieron una evolución favorable, al margen de que fueran diabéticos; sin embargo, los valores promedio fueron siempre superiores en este grupo. Atribuyeron los hallazgos a que los diabéticos están predispuestos en mayor medida a desarrollar enfermedad cardiovascular y tienen peor pronóstico si tienen un infarto agudo de miocardio en comparación con los no diabéticos, y éstos tienen a su vez una peor evolución cuando presentan algún trastorno del metabolismo glucémico en el curso del episodio isquémico ${ }^{3}$.

Además, se logró determinar que las comorbilidades observadas en los pacientes estudiados (diabetes mellitus, hipertensión arterial, tabaquismo y alcoholismo) son estadísticamente diferentes según sea el género y más frecuentes en los hombres. Es conocido que éstos se encuentran establecidos como factores de riesgo cardiovascular y son más frecuentes en hombres y a menor edad. El estudio Rotterdam determinó que, a los 55 años y para los hombres, los riesgos de enfermedad coronaria fueron del $27.2 \%$ contra $16.9 \%$ en mujeres. También se considera el efecto protector estrogénico en las mujeres premenopáusicas, con beneficios antiaterogénicos, los cuales disminuyen de forma progresiva con la edad, lo que retrasaría las consecuencias de la aterosclerosis producidas por la diabetes mellitus y el tabaquismo ${ }^{11}$.

Se estableció que el infarto agudo de miocardio más común es el de elevación del segmento ST y es más frecuente en los hombres. En el ámbito internacional, desde el punto de vista epidemiológico, representa el $25 \%$ de todos los infartos agudos de miocardio y son las mujeres las más afectadas ${ }^{12}$. Sin embargo, se conoce que el infarto miocárdico predomina entre los varones de todos los grupos de edad ${ }^{13}$. En un trabajo de investigación realizado entre 2008 y 2013 con 258 pacientes infartados, se determinó que el género con mayor afectación era el masculino, con una relación $3.4 / 1$ respecto del femenino ${ }^{14}$. Similares resultados se hallaron en un estudio realizado en los Hospitales 
Carlos Andrade Marín y Eugenio Espejo, en el periodo de 2006 a $2007^{15}$. En dicho estudio, el infarto más frecuente, desde el punto de vista topográfico, fue el de la cara inferior en el $43.9 \%$ de los casos. $Y$ al compararlo con el tipo de infarto se observó que los infartos sin elevación del ST predominan en la cara lateral. Esto difiere de los estudios europeos, en los que el infarto predominante es el anterior. Empero, en un estudio publicado en la Revista Latinoamericana de Hipertensión se determinó que la localización topográfica más frecuente del infarto agudo de miocardio era la cara inferior ${ }^{16}$. Sin embargo, existen diferencias en las publicaciones médicas respecto del infarto de miocardio sin elevación del ST y su localización, por lo que podría considerarse que en la población latinoamericana existe una discrepancia en cuanto a la topografía.

El tratamiento clínico fue la conducta inicial más frecuente, con $53.17 \%$ de los casos, y se realizó angioplastia de rescate en el $16.59 \%$. No obstante, las recomendaciones actuales indican que es la intervención coronaria percutánea primaria la primera opción $n^{17}$, lo que denota que las técnicas invasivas no se hallan al alcance las 24 horas.

La angioplastia con colocación de stent se efectuó en un $53.66 \%$ en la arteria descendente anterior, que es el vaso que con mayor frecuencia según las publicaciones médicas es objeto de angioplastia ${ }^{16}$.

La edad promedio de los pacientes estudiados era de 62.99 años, similar a lo descrito en trabajos realizados en hospitales del país ${ }^{14,15}$. No se encontraron diferencias estadísticamente significativas al comparar estancia hospitalaria con tipo de infarto, y se determinó que el promedio de días fue de $8.84 \pm 9.05$ en la unidad de estudio, lo cual se vincula con la presentación de complicaciones.

\section{Conclusiones}

La relación entre la elevación de marcadores inflamatorios, como la proteína $C$ reactiva y las interleucinas, en el contexto del paciente con infarto agudo de miocardio, y un mayor número de complicaciones es directamente proporcional. Sin embargo, estas pruebas tienen como principales limitantes el alto costo y la escasa accesibilidad, sobre todo en los países en vía de desarrollo en los cuales la falta de recursos técnicos y económicos son problemas transcendentales en los servicios de salud. Ante esto se deben considerar alternativas para evaluar de manera temprana las posibles complicaciones que conlleva esta enfermedad no sólo para reducir costos de hospitalización, sino también para valorar la evolución del paciente, de tal modo que puedan establecerse medidas terapéuticas. En esta investigación se logró determinar que un índice leucoglucémico > 656.8 representa un riesgo de 7.89 veces de desarrollar complicaciones en la población estudiada.

\section{Puntos clave}

\section{¿Qué se sabe del tema?}

El índice leucoglucémico es un marcador poco conocido y de fácil disponibilidad y obtención en la analítica sistemática al ingreso del paciente. Se basa en el recuento leucocitario y la glucosa plasmática, sin perder de vita que la hiperglucemia de estrés facilita la patogenia e intensifica el daño isquémico. El recuento de leucocitos se considera el factor predictor independiente de riesgo coronario en pacientes con o sin enfermedad cardiovascular.

\section{¿Qué aporta de nuevo?}

El índice leucoglucémico predice y permite evaluar, de manera temprana, las posibles complicaciones del infarto de miocardio al conocer la evolución del paciente y el riesgo de mortalidad, y al establecer de manera temprana conductas terapéuticas.

\section{Financiamiento}

Este trabajo ha sido financiado por los autores.

\section{Conflicto de intereses}

Los autores manifiestan no presentar conflicto de intereses.

\section{Responsabilidades éticas}

Protección de personas y animales. Los autores declaran que para esta investigación no se han realizado experimentos en seres humanos ni en animales.

Confidencialidad de los datos. Los autores declaran que han seguido los protocolos de su centro de trabajo sobre la publicación de datos de pacientes.

Derecho a la privacidad y consentimiento informado. Los autores declaran que en este artículo no aparecen datos de pacientes. 


\section{Bibliografía}

1. Roffi M, Patrono C, Collet J, Mueller C, Valgimigli M, Andreotti F, et al. Guía ESC 2015 sobre el tratamiento de los síndromes coronarios agudos en pacientes sin elevación persistente del segmento ST. Rev Esp Cardiol. 2015;68(Suppl. 12):e1-e55.

2. Quiroga W, Conci E, Zelaya F, Isa M, Pacheco G, Sala J, et al. Estratificación del riesgo en el infarto agudo de miocardio según el índice leucoglucémico. ¿El "Killip-Kimball" de laboratorio? Rev Fed Arg Cardiol. 2010;39(Suppl. 1):29-34.

3. León-Aliz E, Moreno F, Pérez G, Vega L, Rabassa M. Índice leuco-glucémico en el infarto agudo de miocardio con elevación del ST, un parámetro sencillo y útil en la valoración de las complicaciones. Clín Invest Ateroscl. 2014;26(Suppl. 4):168-75.

4. Galindo G, Galván M, Nellen G, Almeida E. Asociación entre hiperglucemia de estrés y complicaciones intrahospitalarias. Rev Med Inst Mex Seguro Soc. 2015;53(Suppl. 1):6-12.

5. Núñez J, Fácila L, Llàcer A, Sanchís J, Bodí V, Bertomeu V, et al. Valor pronóstico del recuento leucocitario en el infarto agudo de miocardio: mortalidad a largo plazo. Rev Esp Cardiol. 2005;58(Suppl. 6):631-39.

6. Gómez D, Sánchez G, Pérez M, García R, Arguello J, Penaherrera E, et al. Hiperglucemia se asocia a mayor número de desenlaces adversos en individuos latinoamericanos con infarto agudo de miocardio. Clin Investig Arterioscler. 2015;28(Suppl. 1):9-18.

7. Reyes $W$, Tortajada $G$, Varela $G$. Predictores de arritmias ventriculares en el infarto agudo de miocardio. Rev Urug Cardiol. 2014;29(Suppl. 1):122-27.

8. Bono J, Caruso O, Castellano R, Hasbani E, Luciardi H, Macin E, et al. Síndrome coronario agudo sin elevación del segmento ST. Rev Fed Arg Cardiol. 2014;43(Suppl. 1):38-48.
9. Saldarriaga C, Ocampo L, Gómez A, González C, González N. Prognostic accuracy of Grace and Timi scores in patients undergoing percutaneous coronary intervention for non- ST- elevation acute coronary syndrome. Rev Colom Cardiol. 2014;20(Suppl. 3):130-5.

10. Ascaso J. Leuko-glycaemic index in ST elevation acute myocardial in farction, a simple and useful parameter in the predicting complications. Clín Invest Arterioscl. 2014;26(Suppl. 4):159-60.

11. Leening M, Ferket B, Steyerberg E. Sex differences in lifetime risk and first manifestation of cardiovascular disease: prospective population based cohort study. BMJ 2014;349:3-13.

12. Ferreira, I. Epidemiología de la enfermedad coronaria. Rev Esp Cardiol. 2014;67(Suppl. 2):139-44.

13. López E. La hiperglucemia en el síndrome coronario agudo: ¿Objetivo terapéutico o espectador que confiere un mayor riesgo? Rev Esp Cardiol. 2011;211(Suppl. 6):298-300.

14. Sánchez L, Padilla C, Paredes D. Prevalencia de infarto de miocardio y factores asociados en el hospital José Carrasco Arteaga, de la ciudad de Cuenca, durante el período 2008-2013. Cuenca. 2014. Disponible en: http://dspace.ucuenca.edu.ec/bitstream/123456789 / 20218/1/TESIS. pdf. [Acceso 10 Agt 2016].

15. Barros $\mathrm{H}$. Registro piloto del infarto de miocardio en los hospitales Carlos Andrade Marín y Eugenio Espejo de la ciudad de Quito en pacientes ingresados en el período 2006-2007. Quito. 2008. Disponible en: http:// repositorio.usfq.edu.ec/handle/23000 / 500. [Acceso 9 Mar 2016].

16. Arias A, Álvarez A, Juárez U, Damas F, Martínez C. The TIMI risk score for STEMI predicts in-hospital mortality and adverse events in patients undergoing primary angioplasty. Rev Cardiol Mex. 2014;92(Suppl. 2):7-13.

17. O'Gara P, Kushner F, Ascheim D, Casey D, Chung M, Lemos J, et al. 2013 ACCF/AHA Guideline for the management of ST-elevation myocardial infarction: executive summary. Circulation. 2013;127:529-55. 